\title{
Chloroquine use in the Treatment of COVID-19: Systems Biology Report of Common Targets of SARS- CoV-2 and Chloroquine.
}

Serhiy Souchelnytskyi ( $\nabla$ serhiy@qu.edu.qa )

Qatar University https://orcid.org/0000-0001-8243-9276

Nazariy Souchelnytskyi

Uppsala Universitet

\section{Research}

Keywords: COVID-19, SARS-CoV-2, chloroquine, common targets, companion diagnostic, systems biology

Posted Date: November 3rd, 2020

DOI: https://doi.org/10.21203/rs.3.rs-99139/v1

License: (c) (i) This work is licensed under a Creative Commons Attribution 4.0 International License. Read Full License 


\section{Abstract}

BACKGROUND: Chloroquine use for treatment of COVID-19 patients has been under discussion and recommendations have been shifting from positive to caution or non-conclusive. Variability of clinical outputs requires understanding of mechanisms of the differences. Implementation of a companion diagnostic would allow selecting patients who may benefit from the drug. The first line would be markers already used in clinics. Systems biology opens for an opportunity to identify targets common for chloroquine and SARS-CoV-2. These common targets would be candidates for the companion diagnostic.

METHODS: Systemic analysis of molecular mechanisms and markers engaged by chloroquine and SARSCoV-2 virus was performed. The networks of regulatory mechanisms were explored for an intersection and relevance to clinical markers.

RESULTS: Reported here systemic analysis describes the intersection of molecular mechanisms of chloroquine and processes engaged by COVID-19. 266 nodes provide insight into the mechanisms of chloroquine impact on the infection and represent a pool of companion diagnostic markers. As an example, an intersection with the markers of heart arrhythmia retrieved 19 nodes. Thirteen of them were reported in human plasma: levels of albumin, amyloid precursor protein, and endoglin correlate with adverse cardiac effects.

CONCLUSIONS: Reported intersection nodes of SARS-CoV-2 and chloroquine are the candidate markers for companion diagnostic of the chloroquine application. Some of these markers are already used in the clinic and their interpretation may contribute to monitoring for adverse effects of chloroquine.

\section{Background}

The use of chloroquine for the treatment of COVID-19 patients has been under discussion (1-3). To be effective, chloroquine has to act on its targets that would lead to a therapeutic response. To discriminate between responding, non-responding and adverse effects-prone patients, there is a need of a companion diagnostic for chloroquine. Such markers are routine in oncology $(4,5)$. These markers inform clinicians whether a drug would be useful for a given patient. Without these markers, an effect of drugs is frequently non-conclusive when evaluated at the population level. That may explain recent reports of non-conclusive benefit from use of chloroquine and hydroxychloroquine (www.who.int/publications/m/item/targetedupdate-safety-and-efficacy-of-hydroxychloroquine-or-chloroquine-for-treatment-of-covid-19). Chloroquine and hydroxyl chloroquine are used as immunomodulators and showed promising data in in vitro studies of COVID-19 management (1-3). However, the number of clinical evidence is still not sufficient to claim a high certainty conclusion. The systems biology approach may offer the way to identification of markers that would identify patients who may benefit from the drug and those patients who would not. Companion diagnostics with the predicted markers allows selection of responsive patients and prediction of the disease development.

Chloroquine has been used since the 1940th. Studies of this remedy generated information about molecular mechanisms of its action. An international DrugBank depository (www.drugbank.ca) is an example of a 
curated and proven drug target database (6). The studies of COVID-19 are not yet as extensive as studies of chloroquine, but there are already reports of SARS-CoV-2 targets in human cells (7-9). Identified targets reflect molecular mechanisms engaged by chloroquine and COVID-19, and systems biology allows identification of these regulatory processes. A number of network building tools and high-quality databases are available for systemic analysis of molecular mechanisms engaged by COVID-19 and chloroquine (7, 1013). An analysis of regulatory networks is the most comprehensive way to explore mechanisms that are initiated or dependent on the targets of COVID-19 and chloroquine. Comprehensiveness is ensured by the incorporation of the experimental data from hundreds to thousands of reports. For example, UniProt database contains 562,755 records of experimental data (uniprot.org) (14). This a rich source for systemic network analysis.

COVID-19 infection manifests in many different clinical symptoms (15-17). It indicates that the virus employs different molecular mechanisms and attacks different types of cells. Here we report an identification of potential markers to evaluate the efficacy of chloroquine in the treatment of COVID-19 patients. Our systemic analysis identified 266 nodes, i.e. genes and proteins that represent common molecular mechanisms engaged by chloroquine and COVID-19. An example of cardiac arrhythmia showed 19 potential companion diagnostic markers for chloroquine use and prediction of cardiac adverse effects.

\section{Methods}

The datasets for building networks were collected as follows, and are listed in Supplementary Table 1. For chloroquine, the targets were retrieved from the Drug Bank depository (drugbank.ca) (6). For SARS-CoV-2 interacting proteins, 322 interactors were reported by Gordon et al., and ACE2 and TMPRSS2 were used (7, 18). For arrhythmia, markers described by Bose et al. were used (19).

The networks building and analysis was performed in Cytoscape (10). The significance for the inclusion of nodes and edges was set to $p<0.05$. For the building of the networks, we used the UniProt database (14). For extraction of intersections, the "Network Analysis" tool of Cytoscape was used. Statistical significance of network building (inclusion of nodes and confidence of edges) was set on $p<0.05$. BiNGO tool was used for the analysis of affected biological processes. For statistical significance, the level was set at $p<0.05$, and the hypergeometric statistical test was used, with Benjamini and Hochberg false discovery rate correction.

A cross-validation analysis of identified nodes with published reports about their clinical values and a role in physiology was performed. We searched PubMed with the Medical Subject Headings (MeSH) of a node and words "COVID-19", "chloroquine", and "heart". Retrieved publications were scrutinized for information about clinical values of the nodes as markers and for involvement of the nodes in molecular mechanisms and biological processes of relevance for a virus infection, predictive marker value, correlation with clinical outputs and adverse effects, and a role in crucial intracellular regulatory mechanisms, e.g. proliferation, death and differentiation of cells.

\section{Results}




\section{Identification of common targets of SARS-CoV-2 and chloroquine}

For chloroquine, there have been reported 11 direct targets, i.e. GSTA2, TNF, TLR9, GST, HMGB1, GSTM1, CYP2C8, CYP3A4, CYP3A5, CYP2D6 and CYP1A1 (Supplementary Table 1). Chloroquine impact on these targets may lead to engagement of a regulatory network containing 1,336 nodes and 2,526 edges

(Supplementary Fig. 1; Supplementary File 1, network "Chloroquine_UniProt”). The network was built with the retrieval of interaction data from the UniProt database. The same database was used to build networks of angiotensin-converting enzyme 2 (ACE2) and type 2 transmembrane serine protease (TMPRSS2) and SARSCoV-2 interactors that are listed in Supplementary Table 1. The structure of the networks are shown in Supplementary Figs. 2 and 3, and the networks are presented in Supplementary File 1 (networks "Cov_UniProt" and "ACE2TMPRSS2_UniProt"). The ACE2/TMPRSS2 network contains 15 nodes and 19 edges, and the COVID-19 network contains 828 nodes and 1,545 edges. These 3 networks represent molecular mechanisms engaged by chloroquine and SARS-CoV-2 directly or via ACE2-TMPRSS2. Note that the graphical presentation of the networks is to illustrate structure of the networks. Cytoscape Session file (Supplementary File 1) provides access to the networks and allows exploration of the networks, zooming on identifiers, perform selection of sub-networks, clustering and search for biological processes of clinical relevance.

To identify mechanisms shared by COVID-19 and chloroquine, we searched for intersections between these 3 networks. The intersection of the chloroquine and ACE2/TMPRSS2 networks extracted only 2 nodes, i.e. albumin and 14-3-3 zeta/delta. This shows that chloroquine has rather a narrow impact on ACE2 and TMPRSS2-dependent mechanisms. The intersection of the chloroquine and SARS-CoV-2 target networks extracted 266 nodes interconnected by 347 edges (Fig. 1A; Supplementary Table 2, Supplementary File 1, network “Intersection_ChloroqUniProt_CovUniProt_.."). This large number of common nodes indicates a significant molecular cross-talk between chloroquine and COVID-19. One hundred nine of these nodes were also detected in the human plasma (Table 1). These intersections identify mechanisms of chloroquine interference with SARS-CoV-2 action and list potential plasma markers (Fig. 2). The intersection nodes may represent markers of companion diagnostic for chloroquine use. If these nodes are affected in a patient infected with the virus, then the chloroquine prescription may be of help, as chloroquine would markers act on/via these affected nodes.

\section{Covid-19 And Cardiac Arrhythmia Markers}

To evaluate whether the intersection nodes would lead to the identification of clinically relevant markers, we used an example of cardiac arrhythmia. Markers of arrhythmia were used to generate a network (Supplementary Fig. 4). The arrhythmia markers are OPN, ANXA5, GDF15, MPO, LGALS3, TNNT2, TNNI3, ANFB, REN, IL6 and CRP (Supplementary Table 1) (19). The arrhythmia network was explored further for the intersection with common nodes of chloroquine and COVID-19 regulatory mechanisms (Fig. 1B; Supplementary File 1 network “Intersection_Arhythmia_Cov19_..")). There were no edges retrieved between these nodes and amyloid precursor protein was retrieved with 3 different accession numbers. We identified 
19 nodes linking arrhythmia markers to chloroquine and COVID-19 (Table 2). Analysis of these 19 nodes showed an engagement of processes affecting the heart and regulation of cell death and proliferation.

Detection of proteins in serum or plasma suggest their suitability as makers for repeatable sampling by blood collection. We used a database of proteins detected in plasma (http://www.plasmaproteomedatabase.org) and retrieved 13 proteins (Table 2). Then, we searched for reports of clinical applications of these 13 proteins as markers of cardiac conditions. Levels of human serum albumin (ALB), amyloid proteins (APP) and soluble endoglin (ENG) correlate with cardiovascular diseases

(Fig. 2). It has to be noted that these markers have also been associated with general conditions and not only cardiac, e.g. hypoalbuminemia associated with liver and kidney diseases, or had a limited use in clinics, e.g. APP or ENG. Albumin concentration below $10 \mathrm{~g} / \mathrm{L}$ correlates with cardiovascular diseases (20). Levels of amyloid precursor protein (APP) higher than $150 \mathrm{pg} / \mathrm{mL}$ correlate with cardiomyopathy (21). Amyloid-beta $(1-40)$ protein was associated with the incidence of coronary heart failure (22). Two of other identified by us proteins, i.e. microtubule-associated protein tau (MART) and prion protein (PRNP) are also associated with the onset of cellular degeneration (23-25). Endoglin is involved in the development and regulation of vasculature. Elevated levels of soluble endoglin in plasma correlate with enhanced left ventricular filling pressure (26). 14-3-3zeta/delta (YWHAZ) is one of the 10 genes enhanced in ischemic stroke (27).

The systems biology approach allowed us to explore published original experimental data in the search for companion diagnostic markers for chloroquine. Reported here 109 intersection nodes represent a pool of these markers. The example of the search for markers to guide the use of chloroquine and preventing cardiac arrhythmia identified 19 candidates. Four of these were reported to correlate with adverse effects, thus confirming the potential clinical value of our approach. Monitoring of the described here markers may help in preventing severe side effects in COVID-19 patients, even if some of the markers are considered as general, or not-frequently used or even novel. The general (ALB) or not-frequently used (APP, ENG, MART, PRNP and YWHAZ) may be applied in clinics already now, as they are approved as markers. Novel candidate markers from the list of 19 nodes would have to be evaluated in clinical trials, and this work contributes with rationale for such trials.

\section{Discussion}

Systemic network analysis becomes a potent and efficient tool for the investigation of correlations and molecular mechanisms $(8,12,13)$. Well-developed and curated databases contain large volumes of original experimental data. This data are available for analysis with a number of tools. Here, we used Cytoscape that allows retrieval of molecular interactions, functional dependencies, correlation and clinical data (10). Used by us the UniProt database contains more than 500,000 curated entries (14). This rich source of data in combination with the efficient analysis tool, i.e. Cytoscape, leads to unveiling novel dependencies. Two hundred sixty-six nodes common for COVID-19 and chloroquine show an extensive impact of chloroquine on the infection (Figs. 1 and 2; Supplementary Table 2). That may explain the clinical efficacy of chloroquine. However, changes in expression and/or activity of many of these nodes may also have undesirable consequences, leading to adverse effects of chloroquine. The complexity of chloroquine molecular 
mechanisms and differences in representation of these mechanisms in different individuals may lead to different clinical outputs.

This manuscript reports the identification of nodes (genes and proteins) common for SARS-CoV-2 and chloroquine. These interaction nodes may be influenced by both the virus and the drug. Therefore, they would reflect whether and how chloroquine may influence SARS-CoV-2-engaged mechanisms. Such nodes can be potential companion diagnostic markers of chloroquine, even if these markers are known for use for other clinical conditions. As an example of applicability of our data, we report 19 marker candidates for guiding chloroquine treatment of SARS-CoV-2-infected patients and monitoring for cardiac arrhythmia (Table 2). Four of these markers are already known to affect cardiac conditions. The decrease in albumin to concentrations below $10 \mathrm{~g} / \mathrm{L}$ correlates with cardiac adverse effects (20). Albumin levels have been recommended for clinical monitoring of COVID-19 patients $(20,28-31)$. Hypoalbuminemia with the albumin levels lower than $35 \mathrm{~g} / \mathrm{L}$ was associated with the 2-time higher risk of the long-term mortality in heart failure (31). Chloroquine was described as a drug against prion and Alzheimer's diseases (32). Prion protein and amyloid beta peptide are likely to be components of the innate immune system (33). Amyloid-beta protein association with coronary heart disease and amyloidosis-related heart disease was reported $(21,22)$. Identification of amyloid precursor protein, microtubule-associated tau and prion proteins indicate a link of cell damage and degeneration to cardiac conditions.

Similar observations were made for other nodes annotated in Table 2. For example, proliferating cell nuclear antigen (PCNA) level increases in arrhythmia, and when chloroquine has an effect, it prevents PCNA increase (34). CD177 was reported to contribute to blocking atrial fibrillation (35). Chloroquine inhibits autophagy and promotes apoptosis, and METTL2, SHLD3, TP53BP1 are engaged nodes in these processes (Table 2) (3638). Cardiomyocyte proliferation is regulated by another identified node, disabled homolog 2 (Dab2) (39). Dab2 is involved in suppression of apoptosis by Epstein-Barr virus (EBV) (40). Two nodes, mitochondrial antiviral signaling protein (MAVS) and DExD/H-Box Helicase 58 (DDX58) were reported as antiviral proteins $(41,42)$. Inhibition of MAVS expression decreased efficacy of hydroxychloroquine against dengue virus (42). These examples show that the identified nodes have a high probability to be markers for a companion diagnostic. The 19 markers annotated in Table 2 are the example of using the pool of 266 common nodes of COVID-19 and chloroquine. Our report provides a basis for further clinical studies of the potential markers.

Reported by us results can be used in clinical practice already now, as some of identified by us nodes are used in clinical diagnostics, e.g. albumin, or testing is available, even if not-frequently, e.g. soluble endoglin and amyloid precursor protein. These markers are used for non-COVID-19 conditions, and repurposing of their use for COVID-19 patients treated with chloroquine can be applied now. For example, a higher risk of adverse cardiac effects would be indicated by downregulation of albumin and up-regulation of amyloid precursor protein, tau protein, prion protein and soluble endoglin $(21,22,26,43)$.

\section{Conclusion}

Presented here network analysis describes nodes common for SARS-CoV-2 and chloroquine. The common nodes are intersections of molecular mechanisms of the virus and the drug. Having two inputs, these nodes are potential markers of a companion diagnostic of chloroquine for the treatment of COVID-19 patients. 
Some of the intersection nodes, e.g. albumin, soluble endoglin and amyloid precursor protein have records of clinical correlations of their expression and cardiac adverse effects. Other proteins are candidates for companion diagnostic in clinical trials of chloroquine in the treatment of COVID-19 infection.

\section{Abbreviations}

ACE2, angiotensin-converting enzyme 2; TMPRSS2, type 2 transmembrane serine protease; MeSH, Medical Subject Headings; ALB, human serum albumin; APP, amyloid proteins; ENG, soluble endoglin; PCNA, proliferating cell nuclear antigen; MART, microtubule-associated protein tau; PRNP, prion protein; YWHAZ, 143-3zeta/delta; MAVS, mitochondrial antiviral signaling protein; DDX58, DExD/H-box helicase 58; disabled homolog 2 (Dab2); EBV, Epstein-Barr virus.

\section{Declarations}

\section{Ethics approval and consent to participate:}

This study was approved by the ethics committee of the Orotta College of Medicine and Health Sciences and the health facility management division of the Ministry of Health. All patients provided written informed consent to participate in this study.

\section{Consent for Publication:}

Since, it is case report. Consent for publication is not applicable here. Personal or identifying information of study participants is not disclosed in any form in this paper.

\section{Availability of data and materials}

All datasets used for this study are available from corresponding author on reasonable request.

\section{Competing interests}

The authors declare no competing interests.

\section{Funding}

This research did not receive any grant from funding agencies in the public, commercial, or not-for-profit sectors.

\section{Acknowledgements}

We would like to thank the members of the Immunoserology Department at Eritrean National Health Laboratory, Eritrean National Higher Education and Research Institutes, and National Animal and Plant Health Laboratory.

\section{Authors' contributions}


MEH, SMR, YS, IME and FT conceived and designed the study. MEH, SMR, YP and MW analyzed the data and revised the paper. $\mathrm{MEH}$ and SMR wrote the manuscript. All authors read and approved the final manuscript.

\section{References}

1. Funck-Brentano C, Salem JE. Chloroquine or hydroxychloroquine for COVID-19: why might they be hazardous? Lancet 2020;S0140-6736(20)31174-0. doi: 10.1016/S0140-6736(20)31174-0.

2. Hernandez AV, Roman YM, Pasupuleti V, Barboza JJ, White CM. Hydroxychloroquine or Chloroquine for Treatment or Prophylaxis of COVID-19: A Living Systematic Review. Ann Intern Med 2020; Online May 27. doi:10.7326/M20-2496, 2020.

3. Borba MGS, Val FFA, Sampaio VS, Alexandre MAA, Melo GC, et al. CloroCovid-19 Team:Effect of High vs Low Doses of Chloroquine Diphosphate as Adjunctive Therapy for Patients Hospitalized With Severe Acute Respiratory Syndrome Coronavirus 2 (SARS-CoV-2) Infection: A Randomized Clinical Trial. JAMA Netw Open. 2020;3(4):e208857. doi:10.1001/jamanetworkopen.2020.8857.

4. Jørgensen JT. A paradigm shift in biomarker guided oncology drug development. Ann Transl Med. 2019;7(7):148. doi:10.21037/atm.2019.03.36.

5. McLachlan J, George A, Banerjee S. The Current Status of PARP Inhibitors in Ovarian Cancer. Tumori. 2016;102(5):433-40. doi:10.5301/tj.5000558.

6. Wishart DS, Feunang YD, Guo AC, Lo EJ, Marcu A, Grant JR, et al. DrugBank 5.0: a major update to the DrugBank database for 2018. Nucleic Acids Res. 2018;46(D1):D1074-82. doi:10.1093/nar/gkx1037.

7. Gordon GE, Jang GM, Bouhaddou M, Xu J, Obernier K, O’Meara MJ, et al. A SARS-CoV-2-Human ProteinProtein Interaction Map Reveals Drug Targets and Potential Drug Repurposing. 2020; bioRxiv March 27: https://doi.org/10.1101/2020.03.22.002386.

8. Ostaszewski M, Mazein A, Gillespie ME, Kuperstein I, Niarakis A, et al. COVID-19 Disease Map, building a computational repository of SARS-CoV-2 virus-host interaction mechanisms. Sci Data. 2020;7(1):136. doi:10.1038/s41597-020-0477-8.

9. Du M, Cai G, Chen F, Christiani DC, Zhang Z, Wang M. Multi-omics Evaluation of Gastrointestinal and Other Clinical Characteristics of SARS-CoV-2 and COVID-19. Gastroenterology 2020; pii: S00165085(20)30399-1. doi: 10.1053/j.gastro.2020.03.045.

10. Shannon P, Shannon P, Markiel A, Ozier O, Baliga O, Wang NS. JT, et al. Cytoscape: a software environment for integrated models of biomolecular interaction networks. Genome Res. 2003;13(11):2498-504.

11. Souchelnytskyi S, Nera A, Souchelnytskyi N. COVID-19 engages clinical markers for the management of cancer and cancer-relevant regulators of cell proliferation, death, migration and immune response. Sci. Rep. 2020; August.

12. Silverman EK, Schmidt HHHW, Anastasiadou E, Altucci L, Angelini M, Badimon L, et al. Molecular networks in Network Medicine: Development and applications. Wiley Interdiscip Rev Syst Biol Med 2020; e1489. doi:10.1002/wsbm.1489. 
13. Bowen JR, Ferris MT, Suthar MS. Systems biology: A tool for charting the antiviral landscape. Virus Res. 2016;218:2-9. doi:10.1016/j.virusres.2016.01.005.

14. The UniProt Consortium. UniProt: a worldwide hub of protein knowledge. Nucleic Acids Res. 2019;47:D506-15.

15. Extance A. Covid-19 and long term conditions: what if you have cancer, diabetes, or chronic kidney disease? BMJ 2020; Mar 25: 368:m1174. doi: 10.1136/bmj.m1174.

16. Patel KP, Patel PA, Vunnam RR, Hewlett AT, Jain R, Jing R, Vunnam SR. Gastrointestinal, hepatobiliary, and pancreatic manifestations of COVID-19. J Clin Virol. 2020;128:104386. doi:10.1016/j.jcv.2020.104386.

17. Henry BM, Santos de Oliveira MH, Benoit S, Plebani M, Lippi G. Hematologic. Biochemical and Immune Biomarker Abnormalities Associated With Severe Illness and Mortality in Coronavirus Disease 2019 (COVID-19): A Meta-Analysis. Clin Chem Lab Med. 2020;58(7):1021-28.

18. Hoffmann M, Kleine-Weber H, Schroeder S, Krüger N, Herrler T, Erichsen S, et al. SARS-CoV-2 Cell Entry Depends on ACE2 and TMPRSS2 and Is Blocked by a Clinically Proven Protease Inhibitor. Cell. 2020;181(2):271 - 80.e8. doi:10.1016/j.cell.2020.02.052.

19. Bose A, Truong QA, Singh JP. Biomarkers in electrophysiology: role in arrhythmias and resynchronization therapy. J Inter Card Electrophysiol. 2015;43(1):31-44. doi:10.1007/s10840-015-9982-7.

20. Ronit A, Kirkegaard-Klitbo DM, Dohlmann TL, Lundgren J, Sabin CA, Phillips AN, Nordestgaard BG, Afzal S. Plasma albumin and incident cardiovascular disease: results from the CGPS and an updated metaanalysis. Arterioscler Thromb Vasc Biol. 2020;40(2):473-82. doi:10.1161/ATVBAHA.119.313681.

21. Wolfson AW, Shah KS, Patel JK. Amyloid and the heart. Curr Cardiol Rep. 2019;21(12):164. doi:10.1007/s11886-019-1230-9.

22. Stamatelopoulos K, Mueller-Hennessen M, Georgiopoulos G, Sachse M, Boeddinghaus J, Sopova K, et al. Amyloid-beta (1-40) and the Risk of Death From Cardiovascular Causes in Patients With Coronary Heart Disease. J Am Coll Cardiol. 2015;65(9):904-16. doi:10.1016/j.jacc.2014.12.035.

23. Mehrpour M, Codogno P. Prion Protein: From Physiology to Cancer Biology. Cancer Lett. 2010;290(1):123. doi:10.1016/j.canlet.2009.07.009.

24. Galenko O, Jacobs V, Knight S, Bride D, Cutler MJ, Muhlestein JB, Carlquist JL, Anderson JL, Knowlton $\mathrm{KU}$, Bunch J. Circulating Levels of Biomarkers of Cerebral Injury in Patients With Atrial Fibrillation. Am J Cardiol. 2019;124(11):1697-700. doi:10.1016/j.amjcard.2019.08.027.

25. Murakami N, Oyama F, Gu Y, McLennan IS, Nonaka I, Ihara Y. Accumulation of Tau in Autophagic Vacuoles in Chloroquine Myopathy. J Neuropathol Exp Neurol. 1998;57(7):664-73. doi:10.1097/00005072-199807000-00003.

26. Meluzín J, Tomandl J. Can biomarkers help to diagnose early heart failure with preserved ejection fraction? Dis Markers 2015; 426045. doi:10.1155/2015/426045.

27. Eyileten C, Wicik Z, De Rosa S, Mirowska-Guzel D, Soplinska A, Indolfi C, Jastrzebska-Kurkowska I, Czlonkowska A, Postula M. MicroRNAs as Diagnostic and Prognostic Biomarkers in Ischemic Stroke-A Comprehensive Review and Bioinformatic Analysis. Cells 2018;7(12):249. doi: 10.3390/cells7120249. 
28. Kavsak PA, de Wit K, Worster A. Clinical Chemistry Tests for Patients With COVID-19 - Important Caveats for Interpretation. Clin Chem Lab Med. 2020;Apr 16;/j/cclm.ahead-of-print/cclm-2020-0436/cclm-20200436.xml. doi:10.1515/cclm-2020-0436.

29. Kavsak PA, de Wit K, Worster A. Emerging Key Laboratory Tests for Patients With COVID-19. Clin. Biochem. 2020;Apr 30;S0009-9120(20)30391-X. doi: 10.1016/j.clinbiochem.2020.04.009.

30. Suzuki S, Hashizume N, Kanzaki Y, Maruyama T, Kozuka A, Yahikozawa K. Prognostic Significance of Serum Albumin in Patients With Stable Coronary Artery Disease Treated by Percutaneous Coronary Intervention. PLoS One 2019;14(7):e0219044. doi: 10.1371/journal.pone.0219044. eCollection 2019.

31. Ancio A, Allepaerts S, Robinet S, Oury C, Pierard LA, Lancellotti P. Serum Albumin Level and Long-Term Outcome in Acute Heart Failure. Acta Cardiol. 2019;74(6):465-71.

doi:10.1080/00015385.2018.1521557.

32. Gay M, Carato P, Coevoet M, Renault N, Larchanché PE, Barczyk A, Yous S, Buée L, Sergeant N, Melnyk P. New Phenylaniline Derivatives as Modulators of Amyloid Protein Precursor Metabolism. Bioorg Med Chem. 2018;26(8):2151-64. doi:10.1016/j.bmc.2018.03.016.

33. Lathe R, Darlix JL. Prion Protein PRNP. A New Player in Innate Immunity? The Aß Connection. J Alzheimers Dis Rep. 2017;1(1):263-75. doi:10.3233/ADR-170037.

34. Zhang S, Zhu C, Liu Q, Wang W. Effects of Chloroquine on GFAP, PCNA and Cyclin D1 in Hippocampus and Cerebral Cortex of Rats With Seizures Induced by Pentylenetetrazole. J Huazhong Univ Sci Technolog Med Sci. 2005;25(6):625-28.

35. doi: 10.1007/BF02896153.

36. Yue H, Liang W, Gu J, Zhao X, Zhang T, Qin X, Zhu G, Wu Z. Comparative transcriptome analysis to elucidate the therapeutic mechanism of colchicine against atrial fibrillation. Biomed Pharmacother. 2019;119:109422. doi:10.1016/j.biopha.2019.109422.

37. Song H, Feng X, Zhang H, Luo Y, Huang J, Lin M, et al. METTL3 and ALKBH5 Oppositely Regulate M 6 A Modification of TFEB mRNA, Which Dictates the Fate of Hypoxia/Reoxygenation-Treated Cardiomyocytes. Autophagy. 2019;15(8):1419-37. doi:10.1080/15548627.2019.1586246.

38. Noordermeer SM, Adam S, Setiaputra D, Barazas M, Pettitt SJ, Ling AK, et al. The Shieldin Complex Mediates 53BP1-dependent DNA Repair. Nature. 2018;560(7716):117-21. doi:10.1038/s41586-0180340-7.

39. Vucicevic L, Misirkic-Marjanovic M, Paunovic V, Kravic-Stevovic T, Martinovic T, Ciric D, et al. Autophagy inhibition uncovers the neurotoxic action of the antipsychotic drug olanzapine. Autophagy. 2014;10(12):2362-78. doi:10.4161/15548627.2014.984270.

40. Hofsteen P, Robitaille AM, Chapman DP, Moon RT, Murry CE. Quantitative Proteomics Identify DAB2 as a Cardiac Developmental Regulator That Inhibits WNT/ $\beta$-catenin Signaling. Proc. Natl. Acad. Sci. U S A 2016;113(4):1002-7. doi: 10.1073/pnas.1523930113.

41. Min K, Kim JY, Lee SK. Epstein-Barr Virus miR-BART1-3p Suppresses Apoptosis and Promotes Migration of Gastric Carcinoma Cells by Targeting DAB2. Int J Biol Sci. 2020;16(4):694-707. doi:10.7150/ijbs.36595. 
42. Wang R, Zhu Y, Lin X, Ren C, Zhao J, Wang F, Gao X, Xiao R, Zhao L, et al. Influenza M2 Protein Regulates MAVS-mediated Signaling Pathway Through Interacting With MAVS and Increasing ROS Production. Autophagy. 2019;15(7):1163-81. doi:10.1080/15548627.2019.1580089.

43. Wang LF, Lin YS, Huang NC, Yu CY, Tsai WL, Chen JJ, et al. Hydroxychloroquine-inhibited Dengue Virus Is Associated With Host Defense Machinery. J Interferon Cytokine Res. 2015;35(3):143-56. doi:10.1089/jir.2014.0038.

44. Vitverova B, Najmanova I, Vicen M, Tripska K, Igreja Sa IC, et al. Long Term Effects of Soluble Endoglin and Mild Hypercholesterolemia in Mice Hearts. PLoS One. 2020;15(5):e0233725. doi:10.1371/journal.pone.0233725.

\section{Tables}

\section{Table 1}

List of nodes common for COVID-19 and chloroquine that have been observed in the human plasma. These 109 nodes are candidate plasma or serum markers for assessment of chloroquine efficacy in treating COVID19 infection.

At the end of the table are listed 35 nodes that were not observed in the human plasma. 


\begin{tabular}{|c|c|c|}
\hline PPD ID & $\begin{array}{l}\text { Gene } \\
\text { symbol }\end{array}$ & Gene name \\
\hline HPRD_01228 & HMGB1 & high mobility group box 1 \\
\hline HPRD_01456 & PCNA & proliferating cell nuclear antigen \\
\hline HPRD_02717 & NCBP1 & nuclear cap binding protein subunit $1,80 \mathrm{kDa}$ \\
\hline HPRD_01592 & RPS6 & ribosomal protein S6 \\
\hline HPRD_10941 & RPS3 & ribosomal protein S3 \\
\hline HPRD_01245 & NCL & nucleolin \\
\hline HPRD_00883 & HTT & huntingtin \\
\hline HPRD_04323 & TLR2 & toll-like receptor 2 \\
\hline HPRD_02514 & SYK & spleen tyrosine kinase \\
\hline HPRD_03703 & MYD88 & myeloid differentiation primary response 88 \\
\hline HPRD_13847 & MAVS & mitochondrial antiviral signaling protein \\
\hline HPRD_04462 & IKBKB & inhibitor of kappa light polypeptide gene enhancer in B-cells, kinase beta \\
\hline HPRD_00660 & FUS & fused in sarcoma \\
\hline HPRD_01142 & MAPT & microtubule-associated protein tau \\
\hline HPRD_11299 & USE1 & unconventional SNARE in the ER 1 homolog (S. cerevisiae) \\
\hline HPRD_14389 & METTL3 & methyltransferase like 3 \\
\hline HPRD_00087 & PSEN1 & presenilin 1 \\
\hline HPRD_00100 & APP & amyloid beta (A4) precursor protein \\
\hline HPRD_01222 & CD177 & CD177 molecule \\
\hline HPRD_03333 & ATXN1 & ataxin 1 \\
\hline HPRD_08381 & SIRT1 & sirtuin 1 \\
\hline HPRD_02391 & IL2RG & interleukin 2 receptor, gamma \\
\hline HPRD_00989 & IL4 & interleukin 4 \\
\hline HPRD_07259 & RTN4 & reticulon 4 \\
\hline HPRD_04087 & APBB1 & amyloid beta (A4) precursor protein-binding, family B, member 1 (Fe65) \\
\hline HPRD_01861 & TNFRSF1A & tumor necrosis factor receptor superfamily, member $1 \mathrm{~A}$ \\
\hline HPRD_04583 & RIPK1 & receptor (TNFRSF)-interacting serine-threonine kinase 1 \\
\hline HPRD_02739 & CSNK1A1 & casein kinase 1 , alpha 1 \\
\hline HPRD_02217 & IKBKG & inhibitor of kappa light polypeptide gene enhancer in B-cells, kinase \\
\hline
\end{tabular}


gamma

HPRD_05155 PPARGC1A peroxisome proliferator-activated receptor gamma, coactivator 1 alpha HPRD_05258 AKAP8 A kinase (PRKA) anchor protein 8

HPRD_01238 NFKB1 nuclear factor of kappa light polypeptide gene enhancer in B-cells 1

HPRD_02799 CASP3 caspase 3, apoptosis-related cysteine peptidase

HPRD_03538 TRAF2 TNF receptor-associated factor 2

HPRD_03685 PA2G4 proliferation-associated 2G4, 38kDa

HPRD_05521 HDAC2 histone deacetylase 2

HPRD_01453 PRNP prion protein

HPRD_01470 PTPN11 protein tyrosine phosphatase, non-receptor type 11

HPRD_02480 INSM1 insulinoma-associated 1

HPRD_08950 HDAC3 histone deacetylase 3

HPRD_03143 HDAC1 histone deacetylase 1

HPRD_06942 AG01 argonaute RISC catalytic component 1

HPRD_06943 AG02 argonaute RISC catalytic component 2

HPRD_01494 EPHA2 EPH receptor A2

HPRD_09694 TET1 tet methylcytosine dioxygenase 1

HPRD_01242 HNRNPA1 heterogeneous nuclear ribonucleoprotein A1

HPRD_02911 NCOR1 nuclear receptor corepressor 1

HPRD_04078 EP300 E1A binding protein p300

HPRD_07211 NR1H3 nuclear receptor subfamily 1, group $\mathrm{H}$, member 3

HPRD_02660 NR1H2 nuclear receptor subfamily 1, group $\mathrm{H}$, member 2

HPRD_01574 RB1 retinoblastoma 1

HPRD_08406 MYCBP c-myc binding protein

HPRD_09709 DACT1 dishevelled-binding antagonist of beta-catenin 1

HPRD_03382 PRKACA protein kinase, cAMP-dependent, catalytic, alpha

HPRD_01615 DDX5 DEAD (Asp-Glu-Ala-Asp) box helicase 5

HPRD_03402 RPS6KA1 ribosomal protein S6 kinase, 90kDa, polypeptide 1

HPRD_00303 MCM2 minichromosome maintenance complex component 2

HPRD_10641 AKAP8L A kinase (PRKA) anchor protein 8-like 


\begin{tabular}{|c|c|c|}
\hline HPRD_05397 & AURKB & aurora kinase B \\
\hline HPRD_02910 & NCOR2 & nuclear receptor corepressor 2 \\
\hline HPRD_10566 & SSX2IP & synovial sarcoma, $\mathrm{X}$ breakpoint 2 interacting protein \\
\hline HPRD_01484 & PRKAR2A & protein kinase, cAMP-dependent, regulatory, type II, alpha \\
\hline HPRD_11331 & SNX33 & sorting nexin 33 \\
\hline HPRD_12072 & SNX9 & sorting nexin 9 \\
\hline HPRD_02786 & TEC & tec protein tyrosine kinase \\
\hline HPRD_15407 & SNX18 & sorting nexin 18 \\
\hline HPRD_01835 & ZFP36 & ZFP36 ring finger protein \\
\hline HPRD_01235 & NFKBIA & $\begin{array}{l}\text { nuclear factor of kappa light polypeptide gene enhancer in B-cells } \\
\text { inhibitor, alpha }\end{array}$ \\
\hline HPRD_05759 & TNFRSF21 & tumor necrosis factor receptor superfamily, member 21 \\
\hline HPRD_14732 & MoV10 & Mov10, Moloney leukemia virus 10, homolog (mouse) \\
\hline HPRD_05247 & PABPC1 & poly $(A)$ binding protein, cytoplasmic 1 \\
\hline HPRD_04703 & ADAM17 & ADAM metallopeptidase domain 17 \\
\hline HPRD_01903 & ITGAV & integrin, alpha $\mathrm{V}$ \\
\hline HPRD_00628 & ITGB1 & $\begin{array}{l}\text { integrin, beta } 1 \text { (fibronectin receptor, beta polypeptide, antigen CD29 } \\
\text { includes MDF2, MSK12) }\end{array}$ \\
\hline HPRD_05936 & PACSIN1 & protein kinase $C$ and casein kinase substrate in neurons 1 \\
\hline HPRD_05390 & PACSIN2 & protein kinase $\mathrm{C}$ and casein kinase substrate in neurons 2 \\
\hline HPRD_05937 & PACSIN3 & protein kinase $C$ and casein kinase substrate in neurons 3 \\
\hline HPRD_03254 & UPF1 & UPF1 regulator of nonsense transcripts homolog (yeast) \\
\hline HPRD_03570 & NCOA3 & nuclear receptor coactivator 3 \\
\hline HPRD_02534 & CREBBP & CREB binding protein \\
\hline HPRD_03274 & MAD2L1 & MAD2 mitotic arrest deficient-like 1 (yeast) \\
\hline HPRD_04541 & IQGAP1 & IQ motif containing GTPase activating protein 1 \\
\hline HPRD_06343 & LRPPRC & leucine-rich pentatricopeptide repeat containing \\
\hline HPRD_05944 & HDAC9 & histone deacetylase 9 \\
\hline HPRD_01197 & NGFR & nerve growth factor receptor \\
\hline HPRD_00284 & COMT & catechol-O-methyltransferase \\
\hline HPRD_04870 & TANK & TRAF family member-associated NFKB activator \\
\hline
\end{tabular}




\begin{tabular}{|c|c|c|}
\hline HPRD_05367 & CNOT2 & CCR4-NOT transcription complex, subunit 2 \\
\hline HPRD_02698 & FABP4 & fatty acid binding protein 4 , adipocyte \\
\hline HPRD_02811 & $\mathrm{CHUK}$ & conserved helix-loop-helix ubiquitous kinase \\
\hline HPRD_00589 & ESR1 & Estrogen receptor alpha \\
\hline HPRD_01166 & MYOD1 & myogenic differentiation 1 \\
\hline HPRD_01320 & $\mathrm{CBL}$ & Cbl proto-oncogene, E3 ubiquitin protein ligase \\
\hline HPRD_06780 & KAT2B & $\mathrm{K}$ (lysine) acetyltransferase $2 \mathrm{~B}$ \\
\hline HPRD_02557 & TLE1 & transducin-like enhancer of split 1 (E(sp1) homolog, Drosophila) \\
\hline HPRD_03139 & DAB2 & Dab, mitogen-responsive phosphoprotein, homolog 2 (Drosophila) \\
\hline HPRD_13006 & CBX8 & chromobox homolog 8 \\
\hline HPRD_05569 & TP53BP1 & tumor protein p53 binding protein 1 \\
\hline HPRD_00565 & ENG & endoglin \\
\hline HPRD_00279 & CSNK2A2 & casein kinase 2 , alpha prime polypeptide \\
\hline HPRD_00532 & DNMT1 & DNA (cytosine-5-)-methyltransferase 1 \\
\hline HPRD_01812 & TFRC & transferrin receptor $(\mathrm{p} 90, \mathrm{CD} 71)$ \\
\hline HPRD_01296 & RAB8A & RAB8A, member RAS oncogene family \\
\hline HPRD_05373 & MAP4K3 & mitogen-activated protein kinase kinase kinase kinase 3 \\
\hline HPRD_04091 & ADAM9 & ADAM metallopeptidase domain 9 \\
\hline HPRD_00561 & EEF2 & eukaryotic translation elongation factor 2 \\
\hline HPRD_15942 & PPP1CA & protein phosphatase 1 , catalytic subunit, alpha isozyme \\
\hline HPRD_00062 & ALB & albumin \\
\hline HPRD_03183 & YWHAZ & $\begin{array}{l}\text { tyrosine 3-monooxygenase/tryptophan 5-monooxygenase activation } \\
\text { protein, zeta polypeptide }\end{array}$ \\
\hline \multicolumn{3}{|c|}{ Nodes not observed in human plasma. } \\
\hline \multicolumn{3}{|c|}{ These 35 nodes are unlikely to be plasma or serum markers. } \\
\hline \multicolumn{3}{|c|}{$\begin{array}{l}\text { MAD2L2, EMD, IFIH1, RAB39A, Ccnd1, TSPAN12, TSPAN15, P14340, SORBS2, CBX2, CREBZF, TICAM1, } \\
\text { DDX58, JMJJD6, TBK1, AZI2, E7, SHLD3, METTL14, TIr4, MDC1, CREB3, GPS2, TNIP2, P, TSPAN5, } \\
\text { PHLPP1, CSNK2A1, IRF7, CCND1, VACWR196, PTEN, TLR4, NCF1, Dact2. }\end{array}$} \\
\hline
\end{tabular}

\section{Table 2}

19 common nodes for arrhythmia, COVID-19 and chloroquine. 
13 nodes were described in human plasma. 6 nodes at the end of the table are nodes that were not described in human plasma. Figure 2 describes reported information about these nodes as clinical markers.

\begin{tabular}{|lll|}
\hline PPD ID & $\begin{array}{l}\text { Gene } \\
\text { symbol }\end{array}$ & Gene name \\
\hline HPRD_01456 & PCNA & proliferating cell nuclear antigen \\
\hline HPRD_13847 & MAVS & mitochondrial antiviral signaling protein \\
\hline HPRD_01142 & MAPT & microtubule-associated protein tau \\
\hline HPRD_14389 & METTL3 & methyltransferase like 3 \\
\hline HPRD_00100 & APP & amyloid beta (A4) precursor protein \\
\hline HPRD_01222 & CD177 & CD177 \\
\hline HPRD_03333 & ATXN1 & ataxin 1 \\
\hline HPRD_01453 & PRNP & prion protein \\
\hline HPRD_03139 & DAB2 & Dab, mitogen-responsive phosphoprotein, homolog 2 (Drosophila) \\
\hline HPRD_05569 & TP53BP1 & tumor protein p53 binding protein 1 \\
\hline HPRD_00565 & ENG & endoglin \\
\hline HPRD_00062 & ALB & albumin \\
\hline HPRD_03183 & YWHAZ & $\begin{array}{l}\text { tyrosine 3-monooxygenase/tryptophan 5-monooxygenase activation } \\
\text { protein, zeta polypeptide }\end{array}$ \\
\hline Nodes not detected in plasma \\
\hline & MAD2L2 & Mitotic arrest deficient 2-like protein 2 \\
\hline DDX58 & DEAD box protein 58 \\
\hline & ATAD3A & ATPase family AAA domain containing 3A \\
\hline
\end{tabular}

*PPD, Plasma Proteome Database.

\section{Figures}


Figure 1

A 264 nodes, 347 edges

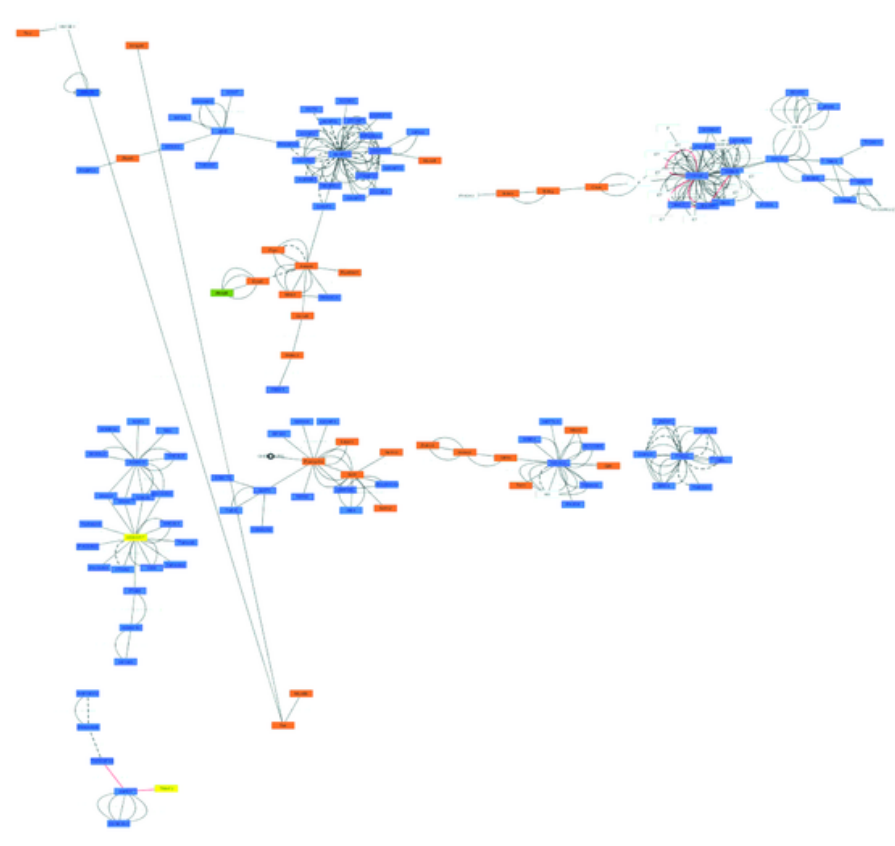

B

19 nodes, no edges

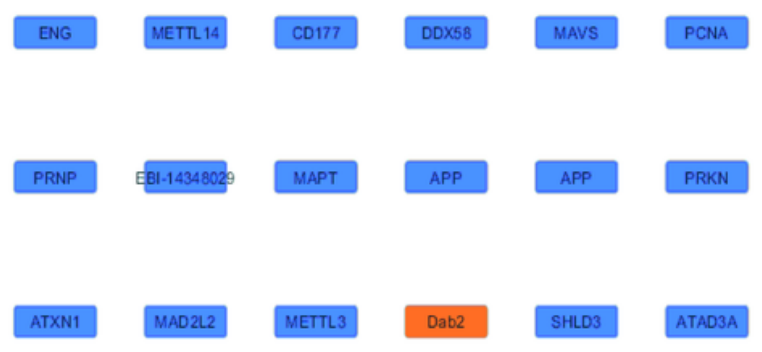

TP538P1

\section{Figure 1}

Structure of the network formed by common targets of chloroquine and COVID-19 (A) and common nodes retrieved by intersection of the networks of markers of arrhythmia and targets of chloroquine and COVID-19 (B) The networks were built with Cytoscape and UniProt database, as described in the text. Numbers of nodes and edges are indicated for (A). Common nodes of arrhythmia markers and targets of chloroquine and COVID-19 did not show connections/edges. The retrieved nodes are shown in (B). The network (A) and nodes (B) are shown to illustrate the structure of the network (A) or absence of it (B). For zooming in the networks for identifiers (nodes and edges identities) and the networks analysis, the networks are in Supplementary File 1 as a Cytoscape Session file (.cys file), available for download at https://figshare.com/articles/online_resource/SupplementaryFileS1_Cytoscape_DataNetwork_cys/12793580 
Figure 2

Common COVID-19 and Chloroquine

(266 nodes)

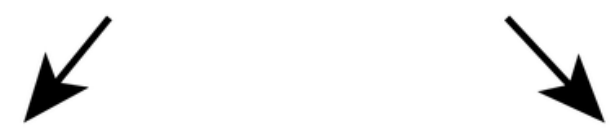

Common arrhythmia/cardiac conditions and COVID-19 and Chloroquine (19 nodes)
Inflammation RNA processing

Transcription Signalling: Translation mTOR/AKT

Proliferation NFkB/IKkB

Cell death
CK2 RPS6K

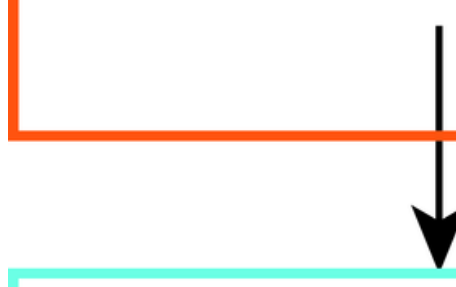

13 nodes described in plasma PCNA, MAVS, MART, METTL3, APP, CD177, ATXN1, PRNP, DAB2, TP53BP1, ENG, ALB, YWHAZ
109 nodes described in plasma

$$
\begin{aligned}
& \checkmark \\
& \text { Albumin }<10 \mathrm{~g} / \mathrm{L} \quad \Rightarrow \text { Cardiovascular mortality/Coronary heart disease } \\
& \text { APP }>150 \mathrm{pg} / \mathrm{mL}=>\text { Amyloid cardiomyopathy } \\
& \text { PRNP, MART } \quad \Rightarrow \text { Cellular degeneration } \\
& \text { Endoglin }(E N G \text {, soluble })=>\text { Cardiac prboblem/Cardiac pressure } \\
& \text { YWHAZ } \quad \Rightarrow \text { Increased in ischemic stroke. }
\end{aligned}
$$

ATXN1, CD177, DAB2, MAVS, METTL3, PCNA, TP53BP1

PCNA, ATAD3A, PRKN, METTL14, SHLD3, DDX58, MAD2L2

\section{Figure 2}

Workflow of selection of potential companion diagnostic markers. Two hundred sixty-six common COVID-19 and chloroquine nodes were evaluated for representation of biological functions and relevance to adverse effects. Retrieved with BiNGO tool biological processes and the nodes of the relevance to the heart arrhythmia markers are annotated.

\section{Supplementary Files}


This is a list of supplementary files associated with this preprint. Click to download.

- 01SupplTableS1.docx

- 01SupplTableS2.docx

- 1SupplFigureS1AB.tif

- 1SupplFigureS2AB.tif

- 1SupplFigureS3AB.tif

- 1SupplFigureS4AB.tif 